\title{
DI Herculis as a test of internal stellar structure and general relativity
}

\section{New apsidal motion rate and evolutionary models}

\author{
A. Claret $^{1}$, G. Torres ${ }^{2}$, and M. Wolf ${ }^{3}$ \\ 1 Instituto de Astrofísica de Andalucía, CSIC, Apartado 3004, 18080 Granada, Spain \\ e-mail: claret@iaa.es \\ 2 Harvard-Smithsonian Center for Astrophysics, 60 Garden St., Cambridge, MA 02138, USA \\ 3 Astronomical Institute, Faculty of Mathematics and Physics, Charles University Prague, 18000 Praha 8, V Holešovičkách 2, \\ Czech Republic
}

Received 22 December 2009 / Accepted 9 February 2010

\begin{abstract}
Context. For the past three decades there has been a serious discrepancy between the observed and theoretical values of the apsidal motion rate $\dot{\omega}$ of the eccentric eclipsing binary DI Her, which has even been interpreted occasionally as a possible failure of general relativity (GR). A number of plausible explanations have been put forward. Recent observations of the Rossiter-McLaughlin effect have shown convincingly that the reason for the anomaly is that the rotational axes of the stars and the orbital axis are misaligned, which changes the predicted rate of precession significantly.

Aims. Although the disagreement is now drastically smaller as a result of those measurements, it formally remains at the level of $50 \%$, owing possibly to errors in the measured apsidal motion rate, outdated stellar models, or inaccuracies in the stellar parameters. The aim of this paper is to address each of these issues to improve the agreement even more.

Methods. New times of minimum have been collected and used for redetermining of the apsidal motion rate. Based on the latest determinations of the absolute dimensions of the binary, we computed new stellar evolution models with updated physical inputs, and derived improved apsidal motion constants for the components. We performed Monte Carlo simulations to infer the theoretical distribution of $\dot{\omega}$, including the contributions from GR, as well as tidal and rotational distortions. All observational errors have been accounted for

Results. Our simulations yield a retrograde apsidal motion rate due to the rotationally-induced oblateness of $-0.00056 \mathrm{deg}^{\mathrm{c} y c l \mathrm{e}^{-1}}$ (mode of the distribution), a GR contribution of $+0.00068 \mathrm{deg}$ cycle $^{-1}$, and a tidal contribution of +0.00034 deg cycle $^{-1}$, leading to a total predicted rate of $+0.00046 \mathrm{deg}$ cycle $^{-1}$. This is in excellent agreement with the newly measured value of $+0.00042 \mathrm{deg} \mathrm{cycle} \mathrm{e}^{-1}$. The formal difference is now reduced to $10 \%$, a small fraction of the observational uncertainties.
\end{abstract}

Key words. binaries: eclipsing - stars: interiors - stars: rotation

\section{Introduction}

For some three decades, the eccentric B-type eclipsing binary DI Her ( $P=10.55$ days) has been known to have an apsidal motion rate that is too slow compared to theoretical predictions (for a brief review, see Claret 1998). Until very recently, this has presented a serious challenge to our understanding of stellar physics, and even the validity of general relativity (GR) has been called into question given that the GR contribution is dominant for DI Her. The discrepancy in the total rate of precession amounts to $400 \%$ or more. This anomaly has persisted even when considering hypothetical stellar models with infinite mass concentration (apsidal motion constant $k_{2}=0$ ), which tends to reduce the predictions, and this was often interpreted as a failure of GR. The problem was all the more puzzling when considering that studies of other binary systems suitable for testing GR and with well-determined absolute dimensions indicated good agreement with theory (see, e.g., Claret 1997; Wolf et al. 2010). This suggested to many that the problem may lie with DI Her itself. A number of hypotheses were put forward over the years to explain the disagreement, including

1. a rapid ongoing circularization of the orbit, affecting the measured times of minimum;

2. the presence of a circumstellar cloud between the components, which would affect the gravitational field of the stars;

3. the presence of a distant third star in the system, which would contribute to the motion of the line of apsides;

4. an alternative theory of gravitation.

Some of these ideas were examined quantitatively and ruled out by Claret (1998). The third body hypothesis has been a serious contender for many years (see, e.g., Khodykin 2007), although no spectroscopic or photometric evidence of it has ever been found. Difficulties in the measurement of such a small apsidal motion as that of DI Her $\left(\dot{\omega}_{\text {obs }}=1.04^{\circ}\right.$ per $100 \mathrm{yr}$, or $0.00030 \mathrm{deg}_{\text {cycle }}{ }^{-1}$; Guinan et al. 1994) were also suggested as a possible explanation from time to time. 
An attractive alternative is a configuration for the binary in which the spin axes are tilted relative to the orbital axis (Shakura 1985; Guinan \& Maloney 1985; Company et al. 1988; Claret 1998). This would introduce a correction to the rotational term of the apsidal motion that can be negative (regression of the line of apsides), resulting in a smaller total $\dot{\omega}$. The young age of the system (a few Myr) certainly makes this plausible, as tidal forces would not have had time to operate and bring the spin axes of the stars into alignment with the axis of the orbit (see Sect. 3). A remarkable confirmation of this prediction has been obtained recently by Albrecht et al. (2009), who provided compelling observational evidence for a peculiar orientation of the stars by taking advantage of the Rossiter-McLaughlin effect. This is a distortion of the spectral lines that occurs during the eclipses, as a result of the partial obscuration of the rotating stellar disks. Using this effect, they were able to measure the angles between the sky projections of the spin axes and the orbital axis, and show that there is indeed a strong misalignment for both components of the binary. It is the first documented case in which this has been shown convincingly for stars in a double-lined eclipsing binary ${ }^{1}$.

With this new information Albrecht et al. (2009) managed to reduce the discrepancy with the predicted apsidal motion rate of DI Her very significantly, so that theory and observation are now in much better agreement. Some of the angles involved in computing the effect of the tilted axes remain unknown, however, so the theoretical prediction necessarily assumes a statistical character in this case, which Albrecht et al. (2009) tackled by means of Monte Carlo simulations. The nominal difference between the measured apsidal motion rate and the mode of their theoretical probability distribution for $\dot{\omega}$ is still about $50 \%$ at present, although the distribution is rather wide so theory and observation are formally consistent with each other. Some of this difference could come from the measurement of $\dot{\omega}_{\text {obs }}$ itself, from inaccuracies in the adopted absolute dimensions of the stars, or from the stellar evolution models used to infer the apsidal motion constants $k_{2}$, which are needed as input to the theoretical calculations.

In this paper we re-examine all the ingredients in order to provide the most accurate predictions and observational constraints. We incorporate new measurements of the eclipse times to derive an improved value of $\dot{\omega}_{\text {obs }}$ for DI Her that is significantly higher than the value adopted by Albrecht et al. (2009). We use the most recent determinations of the mass, radius, and other properties of the stars, and we revisit the temperature determinations. With these we provide new fits to stellar evolution models and derive new apsidal motion constants. We then repeat the comparison of the measured apsidal motion rate with theory, also examining the sensitivity of the predictions to several of the key observables.

\section{Observational data}

\subsection{The absolute dimensions}

The absolute masses and radii of the components of DI Her used here are adopted from the recent compilation by Torres et al. (2009a), in which these and other properties were re-examined or redetermined with a uniform methodology. The uncertainties are adopted from the same source. We summarize all observational results in Table 1 , and describe some of them later. The effective temperatures reported by Torres et al. (2009a) are

\footnotetext{
${ }^{1}$ Spin-orbit misalignment has been reported previously for several host stars of extrasolar transiting planets.
}

Table 1. Observational parameters for DI Her.

\begin{tabular}{lcc}
\hline \hline Parameter & Primary & Secondary \\
\hline Mass $m\left(M_{\odot}\right)$ & $5.17 \pm 0.11$ & $4.524 \pm 0.066$ \\
Radius $R\left(R_{\odot}\right)$ & $2.681 \pm 0.046$ & $2.478 \pm 0.046$ \\
Effective temperature $(\mathrm{K})$ & $17300 \pm 800$ & $15400 \pm 800$ \\
$v \sin \beta\left(\mathrm{km} \mathrm{s}^{-1}\right)$ & $108 \pm 4$ & $116 \pm 4$ \\
$\lambda(\mathrm{deg})$ & $+72 \pm 4$ & $-84 \pm 8$ \\
$\log k_{2}$ & $-2.10 \pm 0.05$ & $-2.13 \pm 0.05$ \\
\hline Orbital period $P($ days) & $10.5501696 \pm 0.0000007$ \\
Reference epoch $T_{0}(\mathrm{HJD})$ & $2447372.9567 \pm 0.0005$ \\
$\omega_{0}$ at reference epoch $(\mathrm{deg})$ & $330.0 \pm 0.1$ \\
Orbital eccentricity $e$ & $0.4895 \pm 0.0008$ \\
Orbital inclination $i(\mathrm{deg})$ & $89.30 \pm 0.07$ \\
$\dot{\omega}_{\text {obs }}($ deg cycle & -1 & $0.00042 \pm 0.00012$ \\
\hline
\end{tabular}

Notes. ${ }^{(*)} P$ corresponds here to the sidereal period $P_{\mathrm{s}}$.

Table 2. New times of minimum light of DI Her.

\begin{tabular}{llccl}
\hline \hline HJD - 2400 000 & Error (days) & Epoch & Filter & Observatory \\
\hline 52456.46107 & 0.0005 & 482.0 & $R$ & Ostrava \\
53511.47699 & 0.0004 & 582.0 & $R$ & Ostrava \\
53933.48379 & 0.0005 & 622.0 & $I$ & Valašské Meziříčí \\
54239.43802 & 0.0008 & 651.0 & $R$ & Ostrava \\
$54627.36687^{*}$ & 0.0005 & 687.5 & $V R I$ & Ostrava \\
\hline
\end{tabular}

Notes. ${ }^{(*)}$ Mean value of VRI measurements.

based on the individual $B-V$ colors as given by Popper (1982) and on his color/temperature relation (Popper 1980). As a check, we used the Strömgren photometry obtained by Hilditch \& Hill (1975) and the calibration of Crawford (1978), and derived a mean de-reddened color for the binary that is essentially identical to Popper's. The color/temperature calibration by Paunzen et al. (2005), which combines several more recent temperature scales, then yields individual temperatures of $17300 \mathrm{~K}$ and $15400 \mathrm{~K}$ for the primary and secondary, with uncertainties of $800 \mathrm{~K}$. These are the temperatures we adopt in this work, which are $300 \mathrm{~K}$ hotter than those listed by Torres et al. (2009a).

\subsection{New apsidal motion rate}

The apsidal motion rate of DI Her was determined by means of an $\mathrm{O}-\mathrm{C}$ diagram analysis. The method we followed is that described by Giménez \& García-Pelayo (1983), which is an iterative weighted least-squares procedure including terms in the eccentricity up to the fifth order. There are five adjustable quantities to be determined: the rate of periastron advance $\dot{\omega}$, the sidereal period $P_{\mathrm{s}}$, the eccentricity $e$, and the longitude of periastron $\omega_{0}$ at the reference epoch $T_{0}$. The periastron position $\omega$ at any epoch $E$ is then given by the linear equation

$\omega=\omega_{0}+\dot{\omega} E$,

and the relation between the sidereal and the anomalistic periods, $P_{\mathrm{s}}$ and $P_{\mathrm{a}}$, is given by

$P_{\mathrm{s}}=P_{\mathrm{a}}\left(1-\dot{\omega} / 360^{\circ}\right)$.

The value for the orbital inclination was adopted as $i=89^{\circ} .30$ from the light-curve analysis of Guinan \& Maloney (1985).

We used all precise photoelectric and CCD timings recently recalculated by Kozyreva \& Bagaev (2009, their Tables 1 and 2), 
A. Claret et al.: DI Herculis as a test of internal stellar structure and general relativity

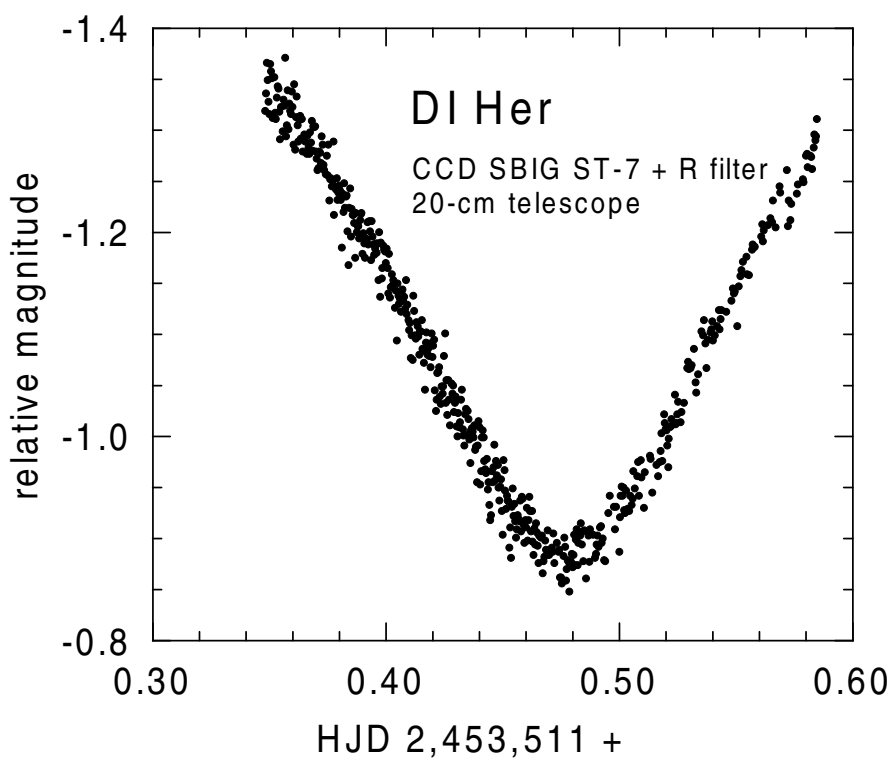

Fig. 1. Differential light curve of DI Her during the primary minimum of 2005 May 20, as measured by Ms. Hana Kučáková at the Johann Palisa Observatory, Technical University Ostrava, Czech Republic. The observations were made through a Johnson $R$ filter with a 20 -cm reflecting telescope and SBIG ST-8XME CCD camera, with exposure times of $25 \mathrm{~s}$. The comparison star was GSC 02109-00247 ( $V=10.04)$. The light curve contains more than 500 points obtained during nearly $6 \mathrm{~h}$ of monitoring.

as well as new eclipse times obtained by us at two observatories with the following instrumentation:

- Observatory and Planetarium of Johann Palisa, Ostrava, Czech Republic: $0.20-\mathrm{m}$ or $0.30-\mathrm{m}$ telescopes with an SBIG ST-8XME CCD camera and Johnson $V, R$, and $I$ filters;

- Valašské Meziříčí Observatory, Czech Republic: 0.30-m Celestron Ultima telescope with an SBIG ST-7 CCD camera and Johnson $R$ filter.

The reduction of all images was performed with the synthetic aperture photometry software developed by Motl (2007). The frames were dark-subtracted and flat-fielded, and the heliocentric correction was applied. An example of these observations is presented in Fig. 1. The new times of primary and secondary eclipse and their errors were determined by the classical Kwee \& van Woerden (1956) algorithm, and are listed in Table 2. Earlier visual and photographic times were not used in our analysis because of the large scatter of those measurements. A total of 69 times of minimum light were employed, including 34 corresponding to the secondary eclipse.

The solution was found by minimizing the standard $\chi^{2}$ sum, and the errors of the observations were adopted as published. The resulting apsidal motion parameters and their uncertainties from the least squares fitting are presented at the bottom of Table 1, and the corresponding $\mathrm{O}-\mathrm{C}$ diagram is shown in Fig. 2. The $\chi^{2}$ value for the best fit was $\chi^{2} \simeq 500$. This is considerably greater than the number of degrees of freedom $(v=64)$, which is most likely due to the formal errors of the eclipse timings being underestimated, as is often found to be the case. The uncertainty of $\dot{\omega}$ reported in Table 1 already accounts for this effect. The extra scatter compared to the uncertainties is seen more clearly in Fig. 3, in which the influence of the apsidal motion terms has been subtracted from the measurements. There is no evidence in

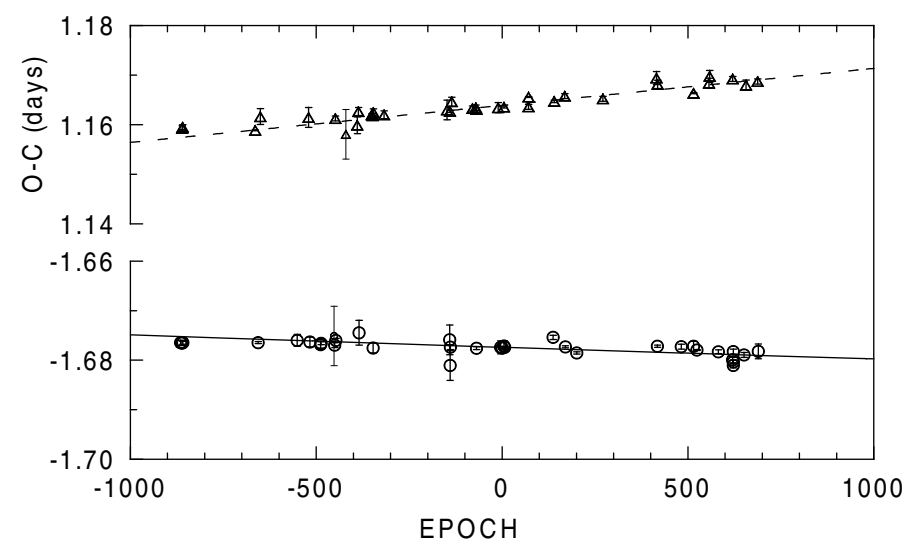

Fig. 2. The O-C diagram of DI Her (eclipse timing residuals), together with our best-fit apsidal motion model. The solid line and circles correspond to the primary, and the dashed line and triangles to the secondary.



Fig. 3. The $\mathrm{O}-\mathrm{C}$ residuals from the photoelectric and $\mathrm{CCD}$ timings of DI Her, after subtraction of the apsidal motion terms. Symbols are as in Fig. 2.

this diagram of any other significant variations, such as might be induced by the presence of a third body in the system.

\section{Stellar evolution models}

As a prerequisite for investigating the theoretical apsidal motion of DI Her, the observed stellar properties such as the mass, radius, and temperature of each component must be well matched by stellar evolution models at a single age for the binary, since these models are then used to infer the apsidal motion constants $k_{2}$ that factor into the rotational and tidal contributions to $\dot{\omega}$. The basic properties of the stellar evolution code used here have been described by Claret (2004). The models include overshooting beyond the formal boundary of the convective core, as determined by the Schwarzschild criterion. The distance over which this is considered to occur is specified as $d_{\text {over }}=\alpha_{\text {ov }} H_{\mathrm{p}}$, where $H_{\mathrm{p}}$ is the pressure scale height taken at the edge of the convective core, as given by Schwarzschild's criterion, and $\alpha_{\mathrm{ov}}$ is a free parameter. We adopt here $\alpha_{\mathrm{ov}}=0.20$, following the results of Claret (2007). The evolution code incorporates radiative opacities from the tables provided by Iglesias \& Rogers (1996), and the calculations of Alexander \& Ferguson (1994) for lower temperatures.

The chemical composition of double-lined eclipsing binaries is often difficult to determine observationally, and that is the case also for DI Her. This introduces some uncertainty in the comparison with stellar evolution models, since the metal 


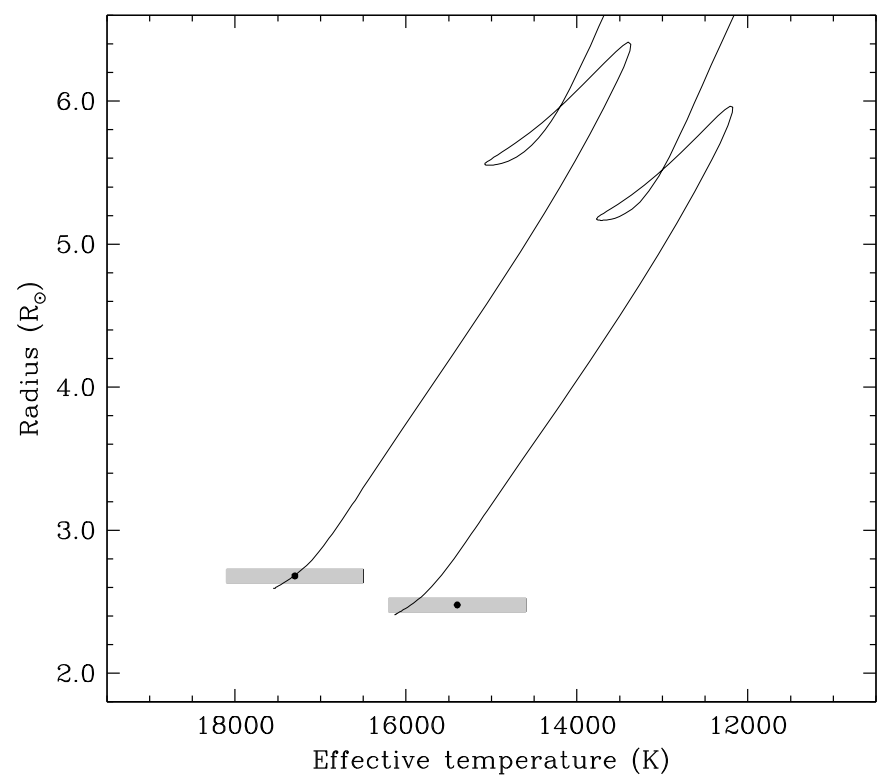

Fig. 4. Stellar evolution models compared with the measurements for DI Her. The evolutionary tracks are computed for the measured masses (see Table 1$)$. The best fit has $(X, Z)=(0.71,0.02)$, implying a chemical composition near solar.

abundance $Z$ then becomes a free parameter. For young systems such as DI Her, $Z$ is quite sensitive to the effective temperatures of the stars. We computed evolutionary tracks for the measured masses and explored a range of compositions, finding the best fit for $(X, Z)=(0.71,0.02)$, which suggests an abundance very near that of the Sun. The observations along with our best fit are displayed in Fig. 4. The agreement with the temperatures is quite satisfactory, given the error bars and other uncertainties described below. Precise age determinations for young systems are not easy to make and require very accurate measurements of the absolute radii. This is illustrated clearly in Fig. 5. As small as the radius errors already are in DI Her $(<2 \%$ relative errors, which are among the best available for eclipsing binaries; see Torres et al. 2009a), the uncertainties allow for a wide range of ages between $\sim 2$ Myr and $\sim 7$ Myr. Nominally the best fit to the radii is achieved at about 4.5 Myr with these models. We note, in passing, that other models such as those from the Yonsei-Yale series (Yi et al. 2001; Demarque et al. 2004) do not yield as good a fit, at least with the grid of standard compositions $(X, Z)$ that are publicly available.

The theoretical apsidal motion constants inferred from our models are $\log k_{21}=-2.10 \pm 0.05$ and $\log k_{22}=-2.13 \pm 0.05$ for the primary and secondary, respectively. These values indicate that the modern models favor stars that are slightly more centrally concentrated in mass than those adopted by Claret (1998) and Albrecht et al. (2009). This goes in the direction of decreasing the predicted apsidal motion rate. Tests were made to evaluate the influence on the inferred $k_{2}$ of uncertainties in the age determination and in the chemical composition, and these uncertainties are included in the errors reported above.

Regarding the measured effective temperatures, it is worth mentioning that because the spin axes of the stars are tilted relative to the orbital axis, the temperature distribution over their disks could be highly non-uniform and asymmetric as viewed from the Earth because of gravity darkening, and it is even possible one or both components are viewed nearly pole-on. There may therefore be a bias in the measured effective temperatures that could amount to several hundred degrees for DI Her, but

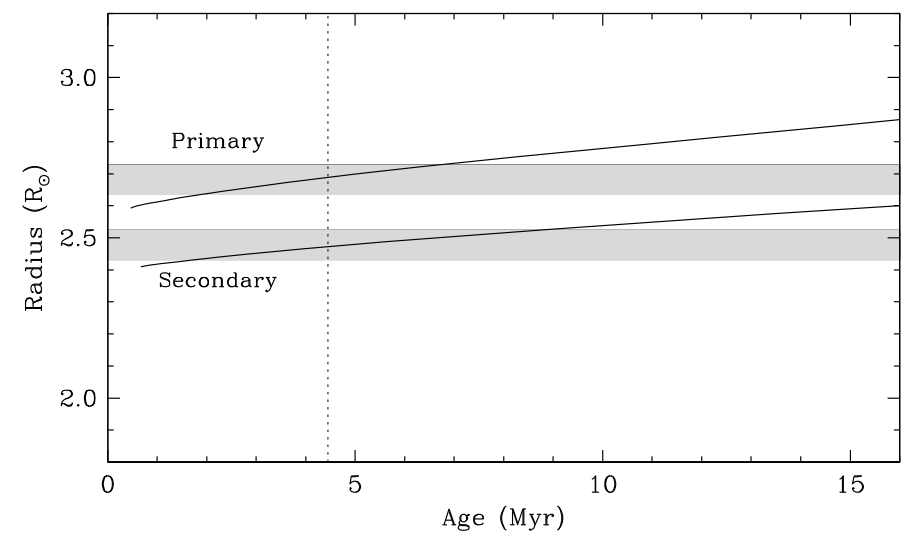

Fig. 5. Radius evolution of DI Her, based on the models shown in Fig. 4. The shaded areas represent the measured radii and their uncertainties. The best-fit age of about 4.5 Myr is indicated by the vertical dotted line.

because the exact orientation is not known (see next section), the effect is difficult to estimate more precisely. Rotation itself changes the internal structure of stars and affects their evolution, generally leading to cooler temperatures. To explore this effect more quantitatively for DI Her we have computed models accounting for rotation (rigid-body approximation), and we find differences of $200 \mathrm{~K}$, depending on the inclination relative to the line of sight. Once again, since the precise orientation of the spin axes is unknown, we are unable to be more specific regarding this effect.

With the models computed in this section we have calculated the age at which the stellar spin axes in DI Her are expected to become aligned with the axis of the orbit due to the action of tidal forces. The procedure follows closely the one described by Torres et al. (2009b, and references therein) for the case of the eclipsing system LV Her. The result using the theory by Zahn (1977, 1989) is $\sim 10^{8}$ yr. With the more general equations by Hut (1981), valid for systems of high eccentricity such as this, we obtain $3 \times 10^{7} \mathrm{yr}$, which is an order of magnitude longer than the evolutionary age. These estimates suggest, as is observed, that tidal forces have not had enough time in this system to make the rotation axes parallel to the axis of the orbit.

\section{The apsidal motion of DI Her}

The total rate of apsidal motion from theory to be compared with the measured value is given by the sum of the individual contributions of each component due to tidal and rotational distortions and the general relativistic term. When accounting for the possibility of misalignment (Shakura 1985), we have

$\dot{\omega}_{\text {total }}=\dot{\omega}_{\mathrm{GR}}+\dot{\omega}_{\mathrm{tidal}, 1}+\dot{\omega}_{\mathrm{tidal}, 2}+\dot{\omega}_{\mathrm{rot}, 1} \phi_{1}+\dot{\omega}_{\mathrm{rot}, 2} \phi_{2}$,

where

$\dot{\omega}_{\text {tidal }, j}=\left[\frac{15 m_{3-j}}{m_{j}} k_{2 j} g(e)\right]\left(\frac{R_{j}}{A}\right)^{5}$,

$\dot{\omega}_{\mathrm{rot}, j}=\left[\left(\frac{\Omega_{j}}{\Omega_{K}}\right)^{2}\left(1+\frac{m_{3-j}}{m_{j}}\right) f(e) k_{2 j}\right]\left(\frac{R_{j}}{A}\right)^{5}$,

$$
\begin{aligned}
\phi_{j}= & -\frac{1}{\sin ^{2} i}\left[\cos \alpha_{j}\left(\cos \alpha_{j}-\cos \beta_{j} \cos i\right)\right. \\
& \left.+\frac{1}{2} \sin ^{2} i\left(1-5 \cos ^{2} \alpha_{j}\right)\right]
\end{aligned}
$$


The subindices "tidal, $j$ " and "rot, $j$ " in Eq. (3) denote the tidal and rotational contributions of component $j$, and "GR" represents the relativistic contribution to the periastron advance. The angle $i$ is the measured inclination of the orbital plane relative to the line of sight (see Table 1), $\alpha_{j}$ are the angles between the rotation axes and the normal to the orbital plane, and $\beta_{j}$ are the angles between the rotation axes and the line of sight to the observer. The symbols $\Omega_{j}$ and $\Omega_{K}$ represent the rotational angular velocity for component $j$ and the Keplerian orbital angular velocity, $A$ is the semimajor axis of the orbit, and $R_{j}$ and $m_{j}$ are the radius and mass of component $j$. The symbols $k_{2 j}$ represent the apsidal motion constant of component $j$, which we determined in the previous section. The auxiliary functions $f(e)$ and $g(e)$ depend only on the eccentricity $e$ and can be written as $f(e)=\left(1-e^{2}\right)^{-2}$ and $g(e)=\left(8+12 e^{2}+e^{4}\right) f(e)^{2.5} / 8$. The factors $\phi_{j}$ capture the dependence on the orientation of the stars. When the spin axes are perfectly aligned with the orbit, $\alpha_{j}=0$, $\beta_{j}=i$, and $\phi_{j}=1$, so that Eq. (3) reduces to the classical expression presented by Sterne (1939a,b).

The relativistic contribution to the apsidal motion, which is independent of stellar structure, was given by Levi-Civita (1937) as

$\dot{\omega}_{\mathrm{GR}}=2.29 \times 10^{-3} \frac{\left(m_{1}+m_{2}\right)}{A\left(1-e^{2}\right)}$ deg cycle ${ }^{-1}$,

where $m_{i}$ and $A$ are in solar units.

By introducing numerical values in Eq. (4), we obtain for the tidal contribution $\dot{\omega}_{\text {tidal }, 1}+\dot{\omega}_{\text {tidal }, 2}=0.000339_{-34}^{+40} \mathrm{deg} \mathrm{cycle}^{-1}$, where the error bars are given in units of the last significant digit and account for all observational uncertainties. This is about $10 \%$ less than obtained by Albrecht et al. (2009), who adopted slightly different absolute dimensions for the stars. The relativistic contribution is $\dot{\omega}_{\mathrm{GR}}=0.000677_{-7}^{+6} \mathrm{deg} \mathrm{cycle}^{-1}$.

The calculation of the rotational contribution is more involved because of to the dependence on the orientation of the system. The angles $\alpha_{j}$ and $\beta_{j}$ in Eq. (6) are not directly measured. However, they are related through the expression

$\cos \alpha_{j}=\cos \beta_{j} \cos i+\sin \beta_{j} \sin i \cos \lambda_{j}$,

in which $\lambda_{j}$ are accessible to observation through the Rossiter-McLaughlin effect and represent the angles between the projections of the spin axes and the orbital axis on the plane of the sky for each star. Albrecht et al. (2009) determined their values to be $\lambda_{1}=+72^{\circ} \pm 4^{\circ}$ and $\lambda_{2}=-84^{\circ} \pm 8^{\circ}$ for the primary and secondary of DI Her, respectively. The angular velocities $\Omega_{j}$ depend on the projected linear rotational velocities, which were measured spectroscopically by Albrecht et al. (2009) as $v_{1} \sin \beta_{1}=108 \pm 4 \mathrm{~km} \mathrm{~s}^{-1}$ and $v_{2} \sin \beta_{2}=116 \pm 4 \mathrm{~km} \mathrm{~s}^{-1}$, and on the unknown inclination angles $\beta_{j}$ needed to de-project the measured velocities in order to infer the equatorial values. We may therefore write

$\frac{\Omega_{j}}{\Omega_{K}}=\frac{P}{2 \pi R_{j}} \frac{\left[v_{j} \sin \beta_{j}\right]}{\sin \beta_{j}}$,

in which we represent $v_{j} \sin \beta_{j}$ in square brackets to indicate that it is a measured quantity.

We follow Albrecht et al. (2009) and perform Monte Carlo simulations in order to determine $\phi_{1}$ and $\phi_{2}$, and with these the total rate of apsidal motion in Eq. (3). The result is a probability distribution rather than a single value. For this we assume that the angles $\beta_{j}$ are distributed completely at random ${ }^{2}$ or, equivalently, that $\cos \beta_{j}$ has a uniform distribution. Knowledge of $\beta_{j}$

\footnotetext{
${ }^{2}$ In other words, $\operatorname{Prob}\left(\beta_{j}\right) \mathrm{d} \beta_{j}=\sin \beta_{j} \mathrm{~d} \beta_{j}$
}

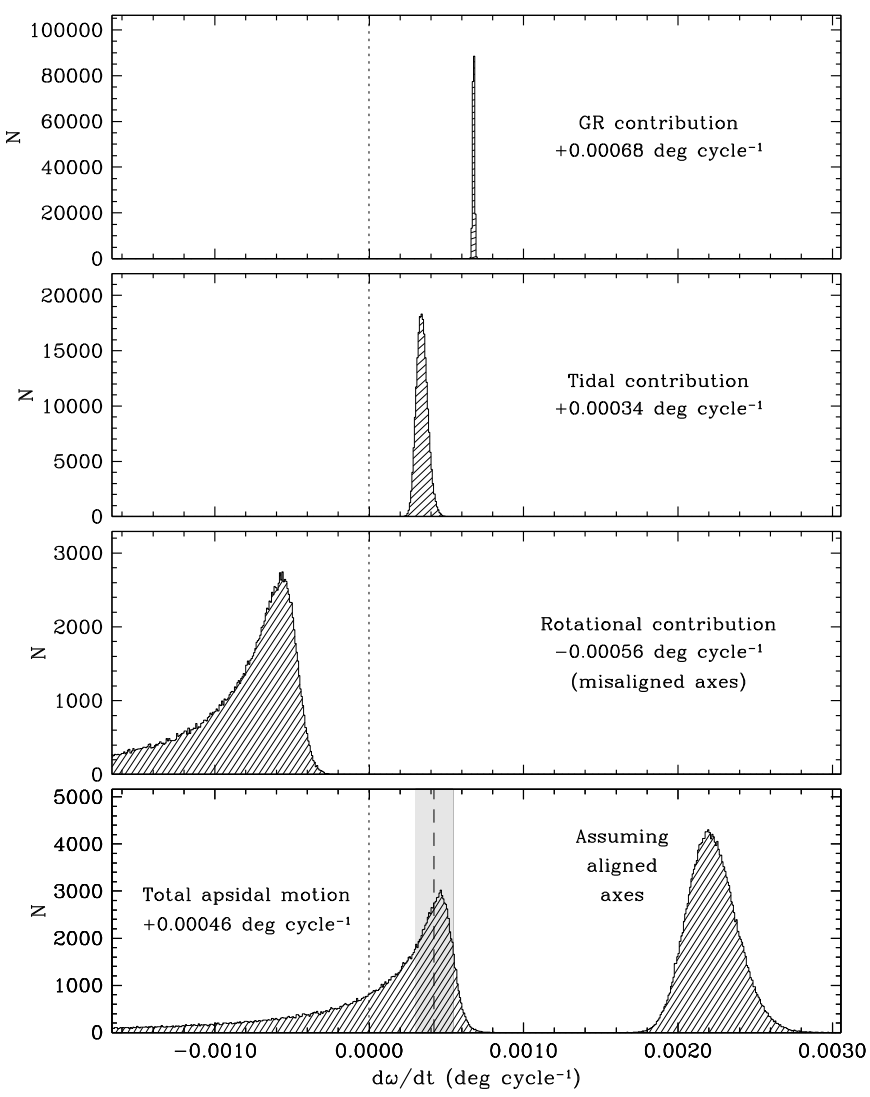

Fig. 6. Monte Carlo calculations for the apsidal motion of DI Her, based on $2 \times 10^{5}$ simulations. From top to bottom, the first three panels show the separate contributions from GR, tidal distortions (2nd and 3rd terms of Eq. (3)), and rotational distortions (4th and 5th terms of Eq. (3)), based on the measurements by Albrecht et al. (2009). The distributions account for all observational errors. The bottom panel shows the predicted total apsidal motion rate on the left (sum of all the above contributions), and on the right the result obtained if the spin axes are assumed to be aligned with the orbital axes, rather than strongly misaligned, as observed. The measured rate of apsidal motion of $\dot{\omega}_{\text {obs }}=$ $0.00042 \pm 0.00012 \mathrm{deg} \mathrm{cycle}^{-1}$ is indicated with the vertical dashed line and shaded area, and agrees remarkably well with the calculation for misaligned axes. The mode of the distribution for the case of aligned axes, on the other hand, is more than 5 times larger than the measured value.

is sufficient to determine $\cos \alpha_{j}$ through Eq. (8), and $\Omega_{j} / \Omega_{K}$ through Eq. (9). As in Albrecht et al. (2009), we exclude angles $\beta_{j}$ that lead to equatorial rotational velocities exceeding the breakup velocity, for which we adopt $600 \mathrm{~km} \mathrm{~s}^{-1}$.

In the first three panels of Fig. 6, we show separately the GR, tidal, and rotational contributions to the apsidal motion rate. The distributions for the first two were also generated with Monte Carlo simulations in which we have assumed that all observational quantities have normally distributed errors. The distribution for the rotational term in the third panel is much wider and asymmetrical, but also sharply peaked, and has a negative modal value of $\dot{\omega}_{\text {rot }}=-0.00056 \mathrm{deg}$ cycle $^{-1}$, as proposed by Shakura (1985) and others, indicating a retrograde motion of the line of apsides due to the misalignment of the spin and orbital axes. This result is numerically some $10 \%$ larger than that obtained by Albrecht et al. (2009), on account of the slightly different absolute dimensions and theoretical $k_{2 j}$ values adopted. The bottom panel of Fig. 6 displays the predicted distribution for the total apsidal motion rate (left), which has a mode of 


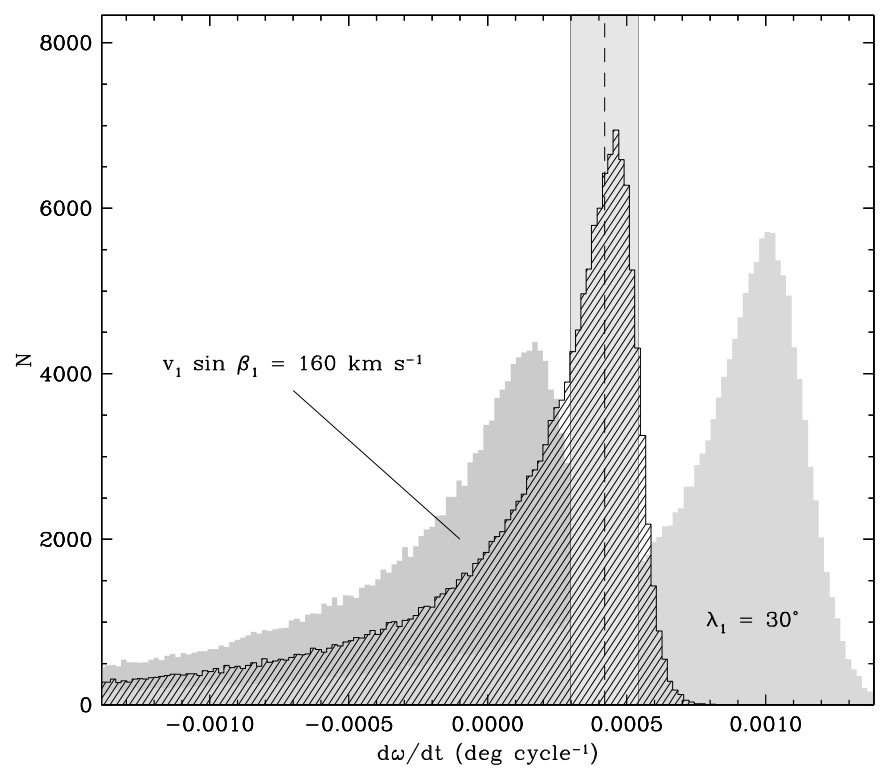

Fig. 7. Sensitivity of the Monte Carlo calculations for the predicted apsidal motion rate of DI Her to changes in some of the input quantities measured with the Rossiter-McLaughlin effect. The hatched histogram is the result using the values exactly as reported by Albrecht et al. (2009), and the vertical dashed line and shaded region marks the new measured apsidal motion rate reported in this work. The shaded histogram on the left is for $v_{1} \sin \beta_{1}=160 \mathrm{~km} \mathrm{~s}^{-1}$ instead of the nominal value of $108 \mathrm{~km} \mathrm{~s}^{-1}$, with all other quantities left at their measured values. The shaded histogram on the right is for $\lambda_{1}=30^{\circ}$ instead of the observed value of $72^{\circ}$. Similar effects are seen when varying those parameters for the secondary.

$\dot{\omega}_{\text {total }}=0.000462_{-473}^{+7}$ deg cycle ${ }^{-1}$ (asymmetric $1 \sigma$ errors enclosing $68.3 \%$ of the results). The agreement with the new measured value of $\omega_{\text {obs }}=0.00042 \pm 0.00012 \mathrm{deg} \mathrm{cycle}^{-1}$ from Sect. 2.2 is excellent, although the theoretical distribution has a long tail toward the left that could certainly accomodate lower values of $\dot{\omega}_{\text {obs. }}$. Still, it is interesting to note that the measurement now agrees much more closely with the most probable value from theory, because the formal difference is only $10 \%$. The improvement compared to the results by Albrecht et al. (2009) is due mostly to the refinement of the measured apsidal motion rate, and to some extent also to the updated absolute dimensions and stellar models leading to more realistic apsidal motion constants. In particular, our models imply stars that are somewhat more centrally concentrated in mass.

If the spin axes of DI Her are assumed to be perfectly aligned with the orbit, instead of strongly misaligned as observed, the resulting Monte Carlo distribution for the total apsidal motion rate is the one shown on the right-hand side of the bottom panel of Fig. 6. The mode of this distribution is $\dot{\omega}_{\text {total }}=0.00220_{-13}^{+18} \mathrm{deg}_{\text {cycle }}{ }^{-1}$, which is 5.2 times greater than the measured value. The distribution is completely inconsistent with the observation. This result for the case of aligned axes is nearly a factor of two more than that presented by Albrecht et al. (2009). The reason for this significant difference is that theirs is based on calculations by Guinan \& Maloney (1985) in which the projected rotational velocities of both stars were taken to be $45 \mathrm{~km} \mathrm{~s}^{-1}$, as measured spectroscopically in the 1970s, whereas we used the values reported by Albrecht et al. (2009) themselves, obtained in 2008, which are considerably higher (Table 1).

The sensitivity of our Monte Carlo calculations for the misaligned case to some of the quantities measured by Albrecht et al. (2009), such as $\lambda_{j}$ and $v_{j} \sin \beta_{j}$, is illustrated in Fig. 7. We show the changes in the resulting distributions when these values are altered one at a time for the primary star, with all other observational quantities held at their nominal values.

The DI Her observations of Albrecht et al. (2009) exploiting the Rossiter-McLaughlin effect represent a breakthrough in our understanding of this system, and the accuracy of their measurements has played a central role in achieving the now excellent agreement with the measured apsidal motion rate of the binary, to within $10 \%$ (a small fraction of the measurement error). The main factor leading to this improvement has been the new measurement of the apsidal motion rate presented here, followed by other refinements including the consideration of stellar models with updated input physics, and more accurate absolute dimensions for the binary components. We now know that the spin axes of the DI Her components are strongly misaligned, and the mystery of its slow apsidal motion rate is solved. However, questions remain. During the process of binary star formation the stellar spins and the orbit presumably derive their angular momentum from the same source, which is the parent molecular cloud. One would then naturally expect that the axes would be closely aligned, yet in this system they are not. Thus, interest in this object is sure to continue as theorists try to answer these questions about DI Her, and observers look for other systems like it.

Acknowledgements. The Spanish MEC (AYA2006-06375) is gratefully acknowledged for its support during the development of this work. G.T. acknowledges partial support through grant AST-0708229 from the US National Science Foundation. The research of M.W. was supported by the Research Program MSM0021620860 of the Ministry of Education of the Czech Republic. We would like to thank Ms. Hana Kučáková, Ostrava Observatory, and Mr. Ladislav Šmelcer, Valašské Meziř́íćí Observatory, for their valuable assistance with the photometric observations, and the anonymous referee for helpful suggestions. This research has made use of the SIMBAD database, operated at CDS, Strasbourg, France, and of NASA's Astrophysics Data System Abstract Service.

\section{References}

Albrecht, S., Reffert, S., Snellen, I. A. G., \& Winn, J. N. 2009, Nature, 461, 373 Alexander, D. R., \& Ferguson, J. W. 1994, ApJ, 437, 879

Claret, A. 1997, A\&A, 327, 11

Claret, A. 1998, A\&A, 330, 533

Claret, A. 2004, A\&A, 424, 919

Claret, A. 2007, A\&A, 475, 1019

Company, R., Portilla, M., \& Giménez, A. 1988, ApJ, 335, 962

Crawford, D. L. 1978, AJ, 83

Demarque, P., Woo, J.-H., Kim, Y.-C., \& Yi, S. K. 2004, ApJS, 155, 667

Giménez, A., \& García-Pelayo, J. M. 1983, Ap\&SS, 92, 203

Guinan, E. F., \& Maloney, F. P. 1985, AJ, 90, 1519

Hilditch, R. W., \& Hill, G. 1975, Mem. R. Astr. Soc., 79, 101

Hut, P. 1981, A\&A, 99, 126

Iglesias, C. A., \& Rogers, F. J. 1996, ApJ, 464, 943

Khodykin, S. A. 2007, IBVS, 5788

Kozyreva, V. S., \& Bagaev, L. A. 2009, Astron. Lett., 35, 483

Kwee, K. K., \& van Woerden, H. 1956, Bull. Astron. Inst. Netherlands, 12, 327

Levi-Civita, T. 1937, Amer. J. Math., 59, 225

Motl, D. 2007, C-MUNIPACK, http: //c-munipack. sourceforge.net

Paunzen, E., Schnell, A., \& Maitzen, H. M. 2005, A\&A, 444, 941

Popper, D. M. 1980, ARA\&A, 18, 115

Popper, D. M. 1982, ApJ, 254, 203

Runkle, R. C. 2003, Ph.D. Thesis, University of North Carolina, unpublished

Shakura, N. I. 1985, Sov. Astr. Lett., 11(4), 225

Sterne, T. E. 1939a, MNRAS, 99, 451

Sterne, T. E. 1939b, MNRAS, 99, 662

Torres, G., Andersen, J., \& Giménez, A. 2009a, A\&A Rev., 18, 67

Torres, G., Lacy, C. H. S., \& Claret, A. 2009b, AJ, 138, 1622

Wolf, M., Claret, A., Kotkova, H., et al. 2010, A\&A, 509, 18

Yi, S. K., Demarque, P., Kim, Y.-C., et al. 2001, ApJS, 136, 417

Zahn, J.-P. 1977, A\&A, 57, 383

Zahn, J.-P. 1989, A\&A, 220, 112 